\title{
Simplicial systems for interval exchange maps and measured foliations
}

\author{
S. P. KERCKHOFF \\ School of Mathematics, The Institute for Advanced Study, \\ Princeton, New Jersey 08540, USA \\ (Received 25 April 1983 and revised 17 October 1983)
}

\begin{abstract}
The spaces of interval exchange maps and measured foliations are considered and an alternative proof that almost all interval exchange maps and measured foliations are uniquely ergodic is given. These spaces are endowed with a refinement process, called a simplicial system, which is studied abstractly and is shown to be normal under a simple assumption. The results follow and thus are a corollary of a more general theorem in a broader setting.
\end{abstract}

\section{Section 0}

An interval exchange map is an orientation-preserving map of the unit interval to itself which is a piecewise continuous isometry defined by choosing $n$ sub-intervals of the unit interval and then interchanging their order by some permutation on $n$ letters. When $n=2$ and the endpoints of the interval are identified, the resulting map is simply a rotation of the circle. The ergodic theory of rotations are wellunderstood; in particular, if the rotation is by an irrational number every point distributes according to Lebesgue measure so that there is a unique Borel probability measure invariant under the map. In such a case, we say the map is uniquely ergodic.

It was fairly quickly discovered ([2], [4]) that when $n>3$ not all interval exchange maps are uniquely ergodic, even in the 'irrational' case, but the examples were very special number theoretically. The expectation (the 'Keane conjecture') was that almost all interval exchange maps were uniquely ergodic (assuming the associated permutation was irreducible). A similar conjecture was made in the case of foliations with transverse measure (see, e.g. [3]). The measured foliations conjecture was solved by Masur [5], the interval exchange version concurrently by Veech [12] and Masur [5], and later an independent proof of both was given by Rees [7].

In this paper we give another proof which is a simplification of an earlier independent proof. Our motivation is two-fold. First, the proof is quite elementary once the basic framework, called a simplicial system, has been set up. It isolates a single, combinatorial property of such a system that must be verified in order for all the results to follow. The results are slightly more general than the previous ones in certain ways (and less so in others - we comment on this throughout the body of the paper). The cases of measured foliations and interval exchange maps can be included together in a single broader setting. Secondly, our approach emphasizes 
the local nature of these results. Whereas the earlier proofs depended on global information like Poincaré recurrence or infinite group actions, the proof given here follows from an elementary measure estimate and the aforementioned combinatorial property. There is some reason to hope that the techniques developed here will be useful elsewhere, e.g. in the study of rational billiards.

Our approach is closest to Veech's. In fact, for the sake of concreteness and familiarity (for some readers, at least) we illustrate our methods with the special case of interval exchange maps using the earlier work of Veech [10] and Rauzy [6] as our point of departure. The basic idea is to describe a transformation on the space of all interval exchange maps $\Sigma$ (the number of intervals and the permutation fixed) which associates to any given exchange map an infinite composition of maps of $\Sigma$ into itself. Topologically, $\Sigma$ is a simplex and the images of the maps are nested sub-simplices whose infinite intersection corresponds to the set of invariant measures on the original exchange map. Thus it suffices to show that almost every such infinite intersection is a single point.

The most direct approach in such a situation is to control the behaviour of the maps of $\Sigma$ into itself, in particular to control the way proportions of measure behave. Once this is done, one gets the normality of the transformation of $\Sigma$ to itself, i.e. the property that every finite block in the sequence of maps that can occur does occur infinitely often for almost every $\lambda \in \Sigma$. From normality all the desired results follow quickly by fairly standard arguments. Specifically, there are maps that always squeeze the diameter of the sub-simplices by a fixed proportion so it follows that the diameter almost always goes to zero and almost all exchange maps (foliations) are uniquely ergodic (theorems 1.10 and 2.3). It also follows easily that the transformation on $\Sigma$ is ergodic and possesses a unique ergodic invariant measure equivalent to Lebesgue measure (theorem 1.12).

As mentioned before, these results are not new, but the proof shows that once the basic set-up of a simplex being mapped into itself over and over via a finite set of rules is established, one need only check a single combinatorial property of the rules to conclude normality (theorem 2.1 ). Furthermore the proof works for certain types of subsets of a simplex; this is illustrated in our proof for the measured foliations case which includes not only the whole space but also certain ('train track') subsets of non-zero codimension. It is in this sense that the results are 'local' and somewhat more general.

§ I deals in detail with interval exchange maps. In $\S$ II we abstract the basic framework and discuss the case of measured foliations.

\section{Section I}

(A) We first recall some results of Veech [10] and Rauzy [6].

Fix a permutation $\sigma$ on $n$ letters and assume that it is not reducible to two disjoint permutations on fewer letters. Subdivide the unit interval $I$ into $n$ sub-intervals of lengths $\lambda_{1}, \ldots, \lambda_{n}$ respectively. Map $I$ to itself by permuting the order of the $n$ intervals in $I$. We call such a map an interval exchange map. For $\sigma$ fixed the space of such maps is parametrized by the $n$-tuples $\lambda_{1}, \ldots, \lambda_{n}$ where $\lambda_{1}+\cdots+\lambda_{n}=1$. 
Thus it is a simplex, denoted by $\Sigma$, which we assume is endowed with Lebesgue measure.

The positive cone of vectors in $\mathbb{R}^{n}$ with non-negative entries is denoted by $\mathbb{R}_{+}^{n}$ and we will use the sum of co-ordinates 'norm'

$$
|x|=\sum_{i=1}^{n} x_{i}, \quad x=\left(x_{1}, \ldots, x_{n}\right)
$$

on $\mathbb{R}_{+}^{n}$. Then $\Sigma$ is embedded in $\mathbb{R}_{+}^{n}$ as the set such that $|x|=1$.

Lebesgue measure is invariant under every interval exchange map and any other invariant measure gives rise to a different exchange map which is topologically conjugate to the original one. If the original map is minimal (every orbit dense) there is an affine isomorphism between the set of invariant Borel measures and the set of topologically conjugate exchange maps. This subset of $\Sigma$ is a sub-simplex of non-zero codimension and can be described as the infinite intersection of nested images of $\Sigma$ under successive projective linear maps.

Definition. Let $A$ be a matrix with non-negative entries. The map $x \mapsto A x /|A x|$ is a projective linear map and will be denoted by $A$.

Maps from $\Sigma$ into itself are defined as follows: fix $\lambda=\left(\lambda_{1}, \ldots, \lambda_{n}\right) \in \Sigma$. Compare the lengths of the last sub-interval and the sub-interval that becomes the last sub-interval, i.e. $\lambda_{n}$ and $\lambda_{\sigma^{-1}(n)}$. If $\lambda_{n}>\lambda_{\sigma^{-1}(n)}$, map $\Sigma$ into itself by the projective linear map determined by the elementary matrix whose only non-zero off-diagonal entry is a ' 1 ' in the $\left(n, \sigma^{-1}(n)\right)$ place. If $\lambda_{\sigma^{-1}(n)}>\lambda_{n}$ the corresponding matrix is just the transpose. (We ignore the set of measure zero where $\lambda_{n}=\lambda_{\sigma^{-1}(n)}$.)

The two images of $\Sigma$ under these maps fill up $\Sigma$ and intersect on the set $\lambda_{n}=\lambda_{\sigma^{-1}(n)}$. The original $\lambda$ is in one of the sub-simplices and we get a new point $\lambda^{(1)}$ in $\Sigma$ under its identification with this sub-simplex.

This operation corresponds to inducing on the sub-interval $[0,1-\beta]$, where $\beta=\lambda_{\sigma^{-1}(n)}$ in the first case, $\lambda_{n}$ in the second. The first return map is again an interval exchange map on $n$ intervals with a new permutation (described below). The set of possibilities for measures of the sub-intervals in the new exchange map, when normalized to have sum equal to 1 , forms a simplex and the projective linear maps above describe the correspondence between the new measures and the original ones. In particular $\lambda^{(1)}$ describes the measures of the sub-intervals of the new interval exchange map.

In order to keep track of how the new intervals relate to the old ones we describe the new permutation by altering either the domain or the range letters, i.e. a 'permutation' means a map from one ordering of $1, \ldots, n$ to another.

If $\lambda_{n}>\lambda_{\sigma^{-1}(n)}$ the image ordering is changed by cyclically moving forward one step those letters occurring after the image of the last letter in the domain. If $\lambda_{\sigma^{-1}(n)}>\lambda_{n}$ the domain is changed by similarly permuting the letters occurring after the one going to the last place. In either case, the lengths to be compared are those of the sub-intervals which are in the $n$th place and are going to the $n$th place. 
Remark. Rather than consider the new permutation as resulting from a re-ordering of the domain set of integers in the second case, Veech [11] and Rauzy [6] choose instead to rename the intervals in the domain. The matrix used is the one above with the columns permuted to keep track of the name changes. The distinction is purely technical.

Applying the same process to each successive $\lambda^{(i)}$, we associate an infinite sequence of elementary matrices and permutations to almost every point $\lambda \in \Sigma$. Denote by $A_{m}$ the product of the first $m$ elementary matrices so that $A_{m+1}=A_{m} E, E$ elementary. Then the $m$ th sub-simplex is just $A_{m} \Sigma$. We think of this sequence as a sequence of symbols where each symbol represents multiplication by a fixed elementary matrix and a change of permutation. Note that there are only finitely many symbols. The sequence of symbols or the sequence $A_{m}$ of matrices will be called the expansion of $\lambda$.

(B) Given this basic setting, our primary goal is to show that the sequence's of symbols are normal, i.e. that any finite block which can occur, does occur infinitely often for almost every $\lambda \in \Sigma$. All our results follow easily from this fact.

Remark. Veech [12] recovers normality as a corollary to his approach.

Since any finite block corresponds to a fixed proportion of the measure of the 'model' simplices corresponding to each permutation and each of the nesting sub-simplices is identified with one such model simplex under the projective linear maps $A_{m}$, one's first attempt would be to show that these maps alter proportions of measure by a bounded amount. Normality would follow easily. Unfortunately there is no bound to the distortion of proportions of measure. However, we will see that in almost every sequence the distortion comes back within a fixed bound infinitely often and that this is sufficient.

To study the distortion of measure we need to compare the Jacobian of the map $A$ at various points $x \in \Sigma$. The following is proved by elementary calculation (see Veech [10]).

LEмMA 1.1. The Jacobian $J_{A}$ of the projective linear map A equals $|A x|^{-n}$, if $A$ is $n \times n$.

Corollary 1.2. If the columns $A_{i}$ of $A$ satisfy $\left|A_{i}\right| /\left|A_{j}\right| \leq M$, then the ratio of the Jacobian of $A$ at two points $x, y \in \Sigma$ is bounded by $M^{n}$. Hence the ratio of the proportion of a subset of $\Sigma$ to the proportion in the image $A \Sigma$ is bounded by $M^{n}$.

Proof of 1.2. $A x=\sum_{i=1}^{n} x_{i} A_{i}$, where $x=\left(x_{1}, \ldots, x_{n}\right)$ and $\left|\sum_{i=1}^{n} x_{i} A_{i}\right|=\sum_{i=1}^{n} x_{i}\left|A_{i}\right|$.

The columns of $A$ are, of course, just the vertices of the image of $\Sigma$ under matrix multiplication by $\boldsymbol{A}$ (without projecting) so we will denote them by $v_{i}$ ( $A$ will be implicit.) We use the terms 'vertices' and 'columns' interchangeably. In light of corollary 1.2 we need to keep track of the ratios of the norms of the $v_{i}$ 's. Since $A_{m+1}=A_{m} E, E$ an elementary matrix, the columns of $A_{i+1}$ are the same as those of $A_{i}$ except that the $k$ th column is added to the $l$ th where the off diagonal 1 in $E$ is in the $(k, l)$ place. We will say that the $k$ th column (vertex) is added to the $l$ th in this case. The first observation is that if $\left|v_{k}\right| \geq\left|v_{l}\right|$ and if $v_{k}$ is added to $v_{l}$, then the 
ratio of the norms is bounded by 2 , independent of the initial ratio. Thus, no matter how badly distributed the norms become, once the 'large' columns are added to the 'small' ones, the ratios are nicely bounded.

In the case of two intervals, there is only one permutation, the matrices are

$$
\alpha=\left(\begin{array}{ll}
1 & 0 \\
1 & 1
\end{array}\right) \text { and } \beta=\left(\begin{array}{ll}
1 & 1 \\
0 & 1
\end{array}\right)
$$

and the powers of $\alpha$ and $\beta$ in the expansion of $\lambda$ are the convergents $a_{i}$ of the continued fraction expansion of

$$
\frac{\lambda_{1}}{\lambda_{1}+\lambda_{2}}=\frac{1}{a_{1}+\frac{1}{a_{2}+\frac{1}{\ldots}}}
$$

After $N \alpha$ 's, say, the proportions of measure are skewed up to a factor of $N^{2}$, e.g. in

$$
\left(\begin{array}{ll}
1 & 0 \\
N & 1
\end{array}\right)
$$

but once this is followed up by a $\beta$ the ratio of the norms of the columns is bounded by 2 , e.g. in

$$
\left(\begin{array}{cc}
1 & 1 \\
N & N+1
\end{array}\right)
$$

As is clear even in this simple case, the ratios of the norms of the columns of the $A_{m}$ will stay bounded in the expansions of only a small set of $\lambda$ 's (those with bounded partial quotients). However, the ratios come back to within a fixed bound infinitely often for a set of $\lambda$ 's of full measure ( $\lambda$ irrational in this case). In the general case this will again be true. The key to the proof will be to 'force' vertices with large norm to be added to ones with small norm.

If the ratios of the norms of some of the columns of $A_{m}$ are large, the measure of $A_{m}(\Sigma)$ in $\Sigma$ will be skewed towards the 'small' vertices. Thus, it will be much more likely that the small columns will be added to the large ones in this case. Fortunately, we don't care how many times columns are added together in certain ways but only how the ratios of the norms are affected. If $\left|v_{i}\right|$ is much smaller than $\left|v_{j}\right|$ then $v_{i}$ will have to be added to $v_{j}$ a large number of times to increase $\left|v_{j}\right|$ by fixed factor. This fact cancels out the propensity of small columns to be added to big ones. This is made precise in proposition 1.3 below.

Proposition 1.3. At any stage in the expansion of $\lambda \in \Sigma$, the probability of a column increasing in norm by a factor of $K$ before being added to another column is less than $n^{2} /(K-1)$.

Proof. Let $v_{1}, \ldots, v_{n}$ be the columns of the matrix $A_{m}$ at the beginning of the block to be considered, i.e. the vertices of the image $V$ of $\Sigma$ under matrix multiplication by $A_{m}$. Then

$$
V=\left\{x \in \mathbb{R}_{+}^{n} \mid x=\sum_{i=1}^{n} \alpha_{i} v_{i}, \quad \sum_{i=1}^{n} \alpha_{i}=1\right\} .
$$


If a column (assume it is $v_{1}$ for concreteness) increases in norm by a factor of $K$ without being added to any other column, then the image of $\lambda$ in $V$ is in the neighbourhood of the face $\alpha_{1}=0$ which is the union of the sets $N_{i}(K), i \neq 1$, defined by

$$
\frac{\alpha_{i}\left|v_{i}\right|}{\alpha_{1}\left|v_{1}\right|} \geq \frac{K-1}{n-1} .
$$

This follows because the contribution to the norm of $v_{1}$ from $v_{i}$ is $c\left|v_{i}\right|$ if $v_{i}$ is added to $v_{1} c$ times, and $v_{i}$ is added to $v_{1} c$ times only if $\alpha_{i} / \alpha_{1} \geq c$. If the norm of $v_{1}$ increases by a factor of $K$, the contribution irom one of the $v_{i}$ is at least $(K-1) /(n-1)\left|v_{1}\right|$.

We need to estimate the proportion of $A_{m}(\Sigma)$ occupied by the projection of this neighbourhood into $A_{m}(\Sigma)$. The projection of $N_{i}(K)$ is the image of $\Sigma$ under the projective linear map given by the matrix $A=A_{m} E$, where $E$ is elementary with its off-diagonal entry in the $(i, 1)$ place equal to $((K-1) /(n-1))\left(\left|v_{1}\right| /\left|v_{i}\right|\right)=\tilde{K}$. Thus the columns of $A$ are the same as those of $A_{m}$ except for the 1st column which equals $v_{1}+\tilde{K} v_{i}$.

It is not hard to compute (see Veech [10]) that the measure of the image of $\Sigma$ under a projective linear map equals $1 /\left(n ! c_{1} c_{2} \cdots c_{n}\right)$ where $c_{i}$ is the norm of the $i$ th column of the matrix determining the map. In our case we get that the ratio of the measure of $A(\Sigma)$ to that of $A_{m}(\Sigma)$ equals:

$$
\frac{n !\left|v_{1}\right| \cdots\left|v_{n}\right|}{n !\left|v_{1}+\tilde{K} v_{i}\right|\left|v_{2}\right| \cdots\left|v_{n}\right|}=\frac{\left|v_{1}\right|}{\left|v_{1}+\tilde{K} v_{i}\right|}<\frac{\left|v_{1}\right|}{\tilde{K}\left|v_{i}\right|}=\frac{n-1}{K-1} \text {. }
$$

Since there are $n-1$ sets, $N_{i}(K)$, the proposition follows.

Remark. The value of the upper bound is unimportant in what follows. All that is needed is that, by choosing $K$ sufficiently large, it can be made less than 1 .

This proposition shows that with fixed probability, independent of the previous part of the expansion, we can assume that a column will be added to some other column before changing norm by too great a factor. Thus some pair of columns will (in almost all expansions) have bounded ratio of norms infinitely often. However, what could conceivably happen is that a subset of the columns will be added amongst themselves over and over without being added to 'outsiders'. This is precisely what happens when the permutation $\sigma$ is reducible. We show that this is impossible when $\sigma$ is irreducible.

Definition. Consider any finite block in the expansion of a point $\lambda \in \Sigma$ and take any subset of two or more vertices of $\Sigma$ such that in that block each member of the subset has been added to some other member and has had some other member added to it. This subset is said to be isolated in that block if none of its members has been added to any vertex outside the subset.

Proposition 1.4. Assume that $\sigma$ is an irreducible interval exchange map. Consider any finite sequence in the expansion of $\lambda \in \Sigma$ starting and ending with the same permutation. Then the only isolated subset of vertices is the entire set. 
Proof. Let $v_{1}, \ldots, v_{k}$ be an isolated subset, $w_{1}, \ldots, w_{m}$ the remaining vertices. The only way a vertex can be added to another vertex or have another one added to it is to be in the $n$th or $\sigma^{-1}(n)$ th position. Once in one of these critical positions, it must remain there or else have the vertex in the other critical position added to it. At some stage two $v_{i}$ 's must occupy both critical positions in order to be added to each other. Any $w_{j}$ in such a position must eventually leave since either it starts out there and must move out to make room for the $v_{i}$ 's or else it must move to get back to the original permutation. In either case, the last $w_{j}$ to leave before two $v_{i}$ 's fill the critical positions must have a $v_{i}$ added to it, which gives a contradiction.

Thus either no $w_{j}$ exists as claimed or none ever enters a critical position. The latter case cannot occur when $\sigma$ is irreducible. This is true because the only way a vertex can move from the $i$ th position to the $n$th is for those in the positions in between to move through the $n$th position first. Similarly, for a vertex whose image under $\sigma$ moves from the $i$ th position to the $n$th position. Since every $v_{i}$ is added to another $v_{j}$, it follows that the positions of all the $v_{i}$ 's and all their images come after those of the $w_{j}$ 's; for any $v_{i}$ occurring before a $w_{j}$ could never be added to another $v_{i}$ without forcing a $w_{j}$ through a critical position as it returned to its original position.

Since the position of the $v_{i}$ 's and their images occur after those of the $w_{j}$ 's, $\sigma$ is reducible, which is a contradiction.

Remark. The non-existence of isolated subsets for blocks beginning and ending at the same permutation is abstracted in the next section as connectedness. It is the only property necessary to prove normality.

We are now in a position to prove the main results.

Definition. A set of vertices $\left\{v_{1}, \ldots, v_{k}\right\}$ is $C$-distributed if $\left|v_{i}\right| /\left|v_{j}\right| \leq C$ for all $i, j$. A simplex is $C$-distributed if its spanning vertices are $C$-distributed.

It will suffice to show that in the expansion of almost every $\lambda \in \Sigma$, the simplex spanned by the columns of $A_{m}$ is $C$-distributed infinitely often for some fixed $C$. The inductive step is the following:

Proposition 1.5. Let $\sigma$ be an irreducible interval exchange map, $A_{m}$ the $m$ 'th matrix in the expansion of $\lambda$. Suppose the collection of columns $v_{1}, \ldots, v_{k}$ is $C^{\prime}$-distributed. Then with probability $\mu^{\prime}>0$, one of the $v_{i}$ 's will be added to a column outside the collection before the maximum of $\left|v_{i}\right|$ increases by a foctor of $K . \mu^{\prime}$ is independent of $A_{m}$, depending only on $C^{\prime}$ and $K$.

Proof. Denote by $w_{j}$ the columns outside the $C^{\prime}$-distributed collection. Call each time the norm of a column $v_{i}$ in the collection increases by a factor of $K^{\prime}$ a jump and assume that $K^{\prime}$ is large enough that

$$
\mu_{K^{\prime}}=1-\frac{n^{2}}{K^{\prime}-1}>0 .
$$

Throughout we assume that each time a $v_{i}$ jumps it has been added to some other column. By proposition 1.3 this occurs with probability greater than $\mu_{K^{\prime}}$. Under this hypothesis, we will see that after a bounded number $(=N)$ of jumps, a $v_{i}$ must have 
been added to a $w_{j}$. Thus this must happen with probability $>\mu_{K^{\prime}}^{N}$ and, letting $K=\left(K^{\prime}\right)^{N}, \mu^{\prime}=\mu_{K}^{N}$, we will be done.

We prove below that after enough jumps some sub-collections of the $v_{i}$ 's must form an isolated collection in that finite block. Since there are only finite number of permutations, only a finite number of such blocks can be strung together before encompassing one beginning and ending with the same permutation. By proposition 1.4 this is impossible; hence some $v_{i}$ must have been added to a $w_{j}$ and proposition 1.5 follows.

First assume that $\left|v_{i}\right|=1, i=1, \ldots, k$.

LEMMA 1.6. There are constants $M_{j}, j=1, \ldots, k$ such that after $M_{j} j u m p s$ either there are $j v_{i}$ 's of norm $K^{\prime}$ or there is an isolated collection of columns. If $j=k$ there is always such a collection.

Proof. The proof is by induction on $j$.

Case $j=2$, $k$ arbitrary. After two jumps, either $\left|v_{1}\right| \geq K^{\prime},\left|v_{2}\right| \geq K^{\prime}$ (we rename columns at will in all that follows) in which case we are done, or $v_{1}$ has jumped twice. In particular, it has been added to something, say $v_{2}$, after it jumped once and its norm was greater than $K^{\prime}$. Thus $\left|v_{2}\right| \geq K^{\prime}$ also. If $j=k=2$ then $v_{1}$ and $v_{2}$ can only be added to each other and are therefore isolated.

$j^{\prime}$ 'th case $\Rightarrow(j+1)$ 'st case, $k$ arbitrary. Assume after $M_{j}$ steps that $\left|v_{i}\right| \geq K^{\prime}, i=1, \ldots, j$ (or else there is an isolated collection in which case we are done). Then during the next $M_{j}$ jumps either $v_{j+1}$ jumps or the first $j v_{i}$ 's are added amongst themselves. (If not, one would be added to $v_{j+1}$ after reaching norm $K^{\prime}$, causing $v_{j+1}$ to jump.) But this is the case $j=k$ so there is an isolated collection.

The general case follows by induction, proving the lemma.

Since we are only interested in increases in norm by factors of $K^{\prime}$, normalizing so that $\max \left|v_{i}\right|=1$ is clearly only a convenience. The further assumption that $\max \left|v_{i}\right| /\left|v_{k}\right|=C^{\prime}=1$ is only slightly more serious.

If $C^{\prime} \neq 1$ (assume max $\left|v_{i}\right|=1$ still), then, when a column increases in norm by a factor of $K^{\prime}$, its norm may only be $K^{\prime} / C^{\prime}$ so that if it is added to a vertex of norm 1 , the latter will increase only by a factor of $K^{\prime} / C^{\prime}$, not $K^{\prime}$. This point can easily be covered by insisting that the first jump a vertex makes be up to norm $K^{\prime}$ not just by a factor of $K^{\prime}$.

Thus isolated collections always appear after a bounded number of jumps unless a $v_{i}$ is added to a $w_{j}$. The proof of proposition 1.5 is completed.

Apply this proposition $n-1$ times, choosing $C^{\prime}=K^{i-1}$ and $\mu_{i}$ the corresponding $\mu^{\prime}$ at the $i$ th stage. Let $C=K^{n-1}$ and $\mu=\prod_{i=1}^{n-1} \mu_{i}$. Then we have:

Corollary 1.7. At any stage of the expansion of $\lambda \in \Sigma$, the columns of $A_{m}$ will become $C$-distributed with probability $\mu$ before the maximum norm of the columns of $A_{m}$ increases by a factor of $K^{n}$. C and $\mu$ are constants depending only on $K$ and $n$.

The set where the columns never become $C$-distributed thus has measure zero. The set where they become $C$-distributed a finite number of times is the countable union of sets of measure zero, hence also has measure zero. 
COROllary 1.8. The columns of $A_{m}$ are $C$-distributed infinitely often in the expansion of almost every $\lambda \in \Sigma, C$ a fixed constant.

COROLlary 1.9. The sequence of symbols in the expansions of an irreducible interval exchange map are normal, i.e. any finite block that can occur does occur infinitely often in the expansion of a.e. $\lambda \in \Sigma$.

Proof. Consider a finite block $B$ and the set where it never occurs. $B$ corresponds to a fixed proportion of the simplex for each permutation and hence to a proportion $\mu$ bounded below in a $C$-distributed simplex. Subdivide $\Sigma$ into $C$-distributed sub-simplices (this is an infinite subdivision) ignoring the set of measure zero where this never occurs. Throw out the subsets of each corresponding to $B$. What is left has measure $1-\mu$. Subdivide the remainder further into $C$-distributed simplices. Throw out the subsets corresponding to $B$ leaving measure $(1-\mu)^{2}$ and so on. The set where $B$ never occurs has measure zero. The set where it appears only finitely many times is a countable union of such sets; hence it also has measure zero.

It is not hard to find a block $B$ within which the diameters of sub-simplices are squeezed by a factor $\neq 1$ independent of its distribution. The following is then immediate:

THEOREM 1.10. For an irreducible interval exchange map, the diameter of the nested sequence of sub-simplices goes to zero in the expansion of almost every $\lambda \in \Sigma$. Hence almost every $\lambda$ is uniquely ergodic.

Remark. Theorem 1.10 in fact follows from normality, given the existence of a single block whose matrix has all positive entries (see [10]).

The shift map on the set of infinite sequences defines a 2 to 1 map $T$ from $\Sigma \times\{\sigma\}$ to itself where $\{\sigma\}$ is the finite collection of permutations occurring in our expansions (called a 'Rauzy class' of permutations).

THEOREM 1.11. The shift map $T: \Sigma \times\{\sigma\} \rightarrow \Sigma \times\{\sigma\}$ is ergodic if $\sigma$ is irreducible.

Proof. Let $A$ be any non-trivial Borel set invariant under $T$. Its complement can be approximated arbitrarily well by a union of sub-simplices which map onto a copy of $\Sigma$ under some power of $T$. By further subdivision find a $C$-distributed sub-simplex in which $A$ has proportion less than $\varepsilon$. Since $A$ is invariant and the maps distort proportions less than $C, A$ must fill up a proportion of the image copy of $\Sigma$ less than $C \varepsilon$. Since $\varepsilon$ is arbitrary, $A$ has measure zero in that copy and since subsets of that copy map onto all the other copies, $A$ has measure zero.

Consider any sub-simplex $B$ of $\Sigma$ which is completely interior to $\Sigma$. Then from lemma 1.1 the ratio of the Jacobians $J_{A}(x) / J_{A}(y)$ is bounded above and below for all $x, y \in B$ independent of $A$. By standard arguments (see [1]) there is a finite measure invariant under the first return map to $B$ which is equivalent to Lebesgue measure. Use this measure to define a measure on $\Sigma \times\{\sigma\}$ invariant under $T$ by the usual inducing process. Since $T$ is ergodic, it will also be equivalent to Lebesgue measure. (It may be infinite however.) 
THEOREM 1.12 (Veech [12]. There is a unique ergodic, $\sigma$-finite measure equivalent to Lebesgue measure invariant under $T: \Sigma \times\{\sigma\} \rightarrow \Sigma \times\{\sigma\}$.

Remark. Finding such a measure is a major step in [12] and in [5].

\section{Section II}

In this section we abstract the content of the first section to what we call a simplicial process and then consider the case of measured foliations.

Consider an $(n-1)$-dimensional simplex $\Sigma$ and a finite discrete set $D$, called the set of states. To almost every point $\lambda \in \Sigma$ associate an infinite sequence of symbols where each symbol represents a projective linear map of $\Sigma$ into itself determined by an elementary matrix and a change from one state to another. To each state is associated a pair $k, l$ of integers between 1 and $n$ which determines which coordinates of $\Sigma$ are to be compared. The simplex $\Sigma$ is mapped into itself by one of the two elementary matrices whose images divide $\Sigma$ into the sets $\lambda_{k}>\lambda_{l}, \lambda_{l}>\lambda_{k}$, depending on which set contains $\lambda$. At the $i$ th stage, the point in $\Sigma$ considered is $\lambda^{(i)}$, the pre-image of $\lambda$ under the product of the first $i-1$ elementary matrices. This association of an infinite sequence of symbols to almost every $\lambda \in \Sigma$ will be called a simplicial process.

Definition. A simplicial process for which there is no non-trivial, isolated collection of vertices in a block beginning and ending with the same state is called connected. ('Isolated' has the same meaning as in section I.)

If every finite block which can occur does occur infinitely often in the expansion of a.e. $\lambda \in \Sigma$, then the simplicial process is called normal. Applying the proofs of the first section, we conclude:

\section{THEOREM 2.1. Every connected simplicial process is normal.}

Hence theorems 1.10-1.12 also follow. In particular, if there is any block whose associated product of elementary matrices has all positive entries, then the infinite intersection of the sub-simplices associated to a.e. $\lambda \in \Sigma$ is a point.

We apply this general situation to prove the corresponding results for the space (and certain subsets of the space) $\mathcal{M F}$ of measured foliations on a surface of genus $g \geq 2$. (The cases $g=0,1$ are trivially handled separately.) Denote by $\mathscr{P} \mathscr{F}$ the space of projective classes of $\mathcal{M F}$, where two foliations are identified if they differ only by multiplying the transverse measure by a scalar. For further details on this space see [9].

A train track $\tau$ is a branched 1-dimensional submanifold of a surface. The complementary regions of $\tau$ are assumed to have Euler characteristic $\chi<0$. (Cusps count as $-\frac{1}{2}$ so, for example, a disk $(\chi=+1)$ with 3 cusps has $\chi=-\frac{1}{2}$. See figure 1 below.) (Details appear in [8].)

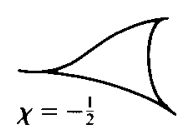

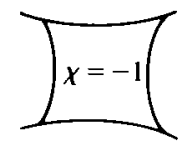

FIGURE 1

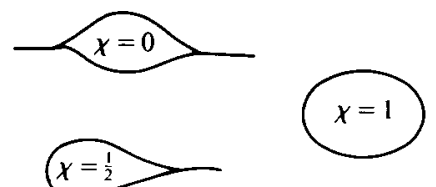

Not Allowed 
Any assignment of non-negative weights to the branches of $\tau$ satisfying the switch condition that the weights coming in equal those coming out

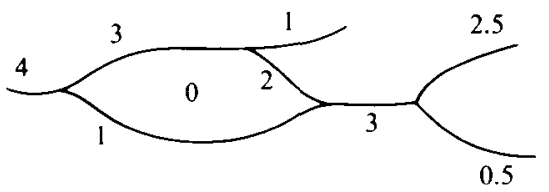

FIGURE 2

determines a measured foliation by running groups of leaves along the branches and assigning them the corresponding transverse measure. (The rest of the surface is filled in by a canonical process, see [8].) The Euler characteristic condition implies that no two such assignments give the same foliation. A foliation is said to be carried by $\tau$ if it can be so described. If all of the complementary regions are triangles, then $\tau$ is called complete; it carries a full dimensional (closed) neighbourhood in $\mathcal{M F}$ (or, projectively, $\mathscr{P} \mathscr{F}$ ).

The given transverse Lebesgue measure of the foliation is invariant by definition and we will say the foliation is uniquely ergodic if this is the only invariant transverse measure (up to projective equivalence). As in the interval exchange case, when the foliation is minimal, there is a 1-1 correspondence between transverse measures and topologically conjugate foliations in $\mathscr{M} \mathscr{F}$.

The set of all weights (projectivized) on the branches describes a simplex but, because of the switch condition, the set of all allowable weights is only a proper subset. The subset may be a (lower-dimensional) simplex but generally is just a polyhedron. Thus we need to modify our proof to cover the case of certain types of subsets preserved under the simplicial process described below.

Remark. It is possible to cover $\mathscr{P} \mathscr{F}$ with simplicial neighbourhoods and modify the simplicial process below to preserve the simplicial structure, but several technical problems arise. These problems are avoided by the approach chosen here which also illustrates a method for adapting the techniques of $\S$ I to proper subsets.

The basic operation on train tracks is the following 'sliding' (or 'splitting') operation. The inverse operation first appears in [8]. Given the local picture in figure 3:

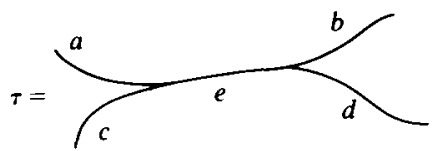

FIGURE 3

alter it as in figure 4:
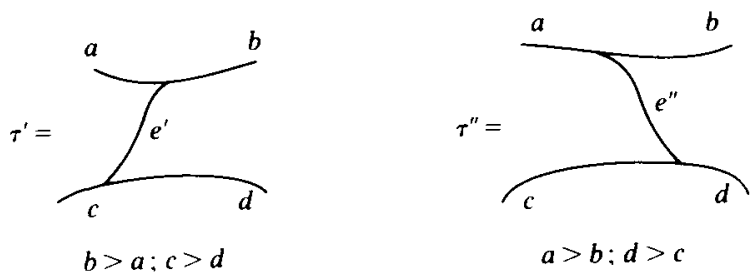

FIGURE 4 
The weights of the new train tracks $\tau^{\prime}, \tau^{\prime \prime}$ are related to the old by the formulae:

$$
\begin{aligned}
& e=e^{\prime}+a+d \\
& e=e^{\prime \prime}+b+c .
\end{aligned}
$$

Thus the corresponding new simplices are mapped into the old by projective linear maps induced by a product of two elementary matrices. We will think of $\tau^{\prime}$ and $\tau^{\prime \prime}$ as embedded in a regular neighbourhood of $\tau$; the formulae above are realized geometrically by collapsing the new train tracks onto $\tau$. The switch conditions are preserved under these maps and the two image simplices divide the original subset satisfying the switch conditions into two pieces. This will be our simplicial system associating an infinite sequence of symbols to almost every foliation carried by $\tau$. The states are the combinatorial types of train tracks (together with a numbering of the branches) which are easily seen to be finite in number. That the matrices are products of two elementary matrices will be discussed below in the proof of theorem 2.3. There is also a choice of which switch to operate on, but any choice will do. To make the process well-defined simply choose, once and for all, a switch for each combinatorial type of train track. At each stage the choice of which way to alter the train track is determined by the topological condition of whether the foliation has a leaf going from ' $b$ ' to ' $c$ ' or from ' $a$ ' to ' $d$ '. Only one of the two can occur and since each subdivision is a topological condition on the foliation, the infinite intersection of these nested sets will contain the topological conjugacy class of $\lambda \in \Sigma$. On the other hand the columns of the product matrices $A_{m}$ in the expansion of $\lambda$ describe the relative frequencies a branch of the $m$ th train track passes through the original branches. As in the case of interval exchange maps (see [10]) any cluster point will determine an invariant measure. Thus the infinite intersection again equals the set of invariant measures.

The only point in the proof of theorem 2.1 where we used the fact that the whole simplex was being considered rather some lower dimensional subset was in proposition 1.3. It was necessary there to show that a fixed proportion of the measure of the simplex was outside certain neighbourhoods of the faces independent of the distribution of the measure. (The neighbourhoods were where a column increased in norm by a factor of $K$ without being added to any other column.) In our case this still remains true for sufficiently small neighbourhoods since there are only finitely many train tracks, hence only finitely many types of subsets satisfying switch conditions and each has some fixed proportion outside such neighbourhoods. By finiteness, a universal proportion is outside these neighbourhoods.

To complete the proof of normality for this system it suffices to investigate connectedness.

Definition. A train track is connected if a dense set of the foliations it carries are minimal. In particular, any complete train track is connected.

If a train track is not connected as a topological space then it is not connected in the sense above. Moreover, if during the splitting process the track becomes disconnected it also is not connected. That the latter doesn't occur is essentially equivalent to connectedness; in particular, theorem 2.3 below says that either an open subset 
of foliations carried by a train track fail to be uniquely ergodic or else almost all of them are.

PROPOSITION 2.2. The simplicial process for a connected train track is connected.

Proof. Suppose there is an isolated collection of vertices $\left\{v_{i}\right\}$ for a block beginning and ending with the same train track $\tau$. During this block no $v_{i}$ has been added to one of the 'outside' vertices $\left\{w_{j}\right\}$ so the closure of the subsimplex corresponding to this block will contain points where the weights are all zero for the $v_{i}$ 's and non-zero for the $w_{j}$ 's. Thus no $w_{j}$ can lead into a $v_{i}$ or else, by the switch condition, it would have to have weight zero.

Recall the configuration for the basic move consisting of a central branch and 4 'wing' branches. Suppose a $v_{i}$ leads into a $w_{j}$. Since this $v_{i}$ must become a centre at some stage in order to be added to something else, it must first be a wing with this $w_{j}$ as centre. It must slide across the $w_{j}$ or else the $w_{j}$ would lead into it at the next stage (figure $5(\mathrm{a})$ ). Thus the $v_{i}$ must slide along $w_{j}$ 's until it comes to another $v_{i}$. (This must occur for the $v_{i}$ to have another $v_{i}$ added to it.) But then the last $w_{j}$ it slid across would lead into this new $v_{i}$ (figure $5(\mathrm{~b})$ ). It follows that no $v_{i}$ can lead into a $w_{j}$ so the $v_{i}$ 's and the $w_{j}$ 's must be disjoint. This is impossible since $\tau$ is connected.

(a)

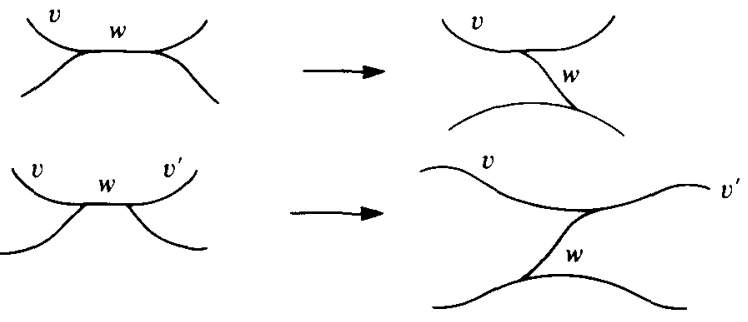

Figure 5

THEOREM 2.3. Almost every measured foliation carried by a connected train track is uniquely ergodic.

Since the space $\mathcal{M F}$ of measured foliations can be covered by finitely many complete (hence connected) train tracks we have:

Corollary 2.4 (Masur [5], see also Rees [7]). Almost every measured foliation is uniquely ergodic.

Proof of theorem 2.3. If each step in the process were multiplication by a single elementary matrix instead of two, the theorem would follow from theorem 2.1 and the earlier comments about the validity of the estimates in proposition 1.3 in this case. However, it does imply that if we break each step in two, almost every expansion becomes $C$-distributed infinitely often. Multiplication by a single elementary matrix will alter this by only a bounded factor so it still holds for a new constant and the theorem follows.

The mapping class group of diffeomorphisms of a surface of genus $g$ (up to isotopy) acts on $\mathscr{P} \mathscr{F}$ and there are only a finite number of equivalence classes of train tracks under this action. We have, similar to theorem 1.11: 
COROllaRy 2.5 (Masur [5], Rees [7]). The mapping class group group acts ergodically on $\mathscr{P} \mathscr{F}$.

Proof. Suppose there were a non-trivial invariant set $A$. Then it would be non-trivial when intersected with a neighbourhood determined by some complete train track. For if it had measure 1 in such a neighbourhood and measure zero in an adjacent one, we could enlarge the first neighbourhood by the inverse of the splitting process so as to intersect the second non-trivially.

As in the proof of theorem 1.11 find a $C$-distributed image of $\Sigma$ in which $A$ has proportion $\varepsilon$. Since there are only finitely many equivalence classes we may assume the map is induced by the action of an element of the mapping class group. $A$ is invariant so it must have proportion at most $C E$ in $\Sigma$. Since $\varepsilon$ is arbitrary, $A$ has measure zero.

Remark. Masur [5] shows that, in fact, the action of the mapping class group on $\mathscr{P} \mathscr{F} \times \mathscr{P} \mathscr{F}$ is ergodic.

Finally, we bring the discussion full circle by suggesting how the operations on the interval exchange maps given in $\S$ I can be interpreted as operations on train tracks. Thus the results of $\S \mathrm{I}$ are direct corollaries of those of $\S$ II.

Form a track with one massive switch with $n$ arcs coming in on the left and $n$ arcs leaving on the right. Attach the arcs on the right to those on the left according to the permutation $\sigma$ (see figure 6) and embed the complex in a surface as a train track. If $\sigma$ is irreducible it will be connected.

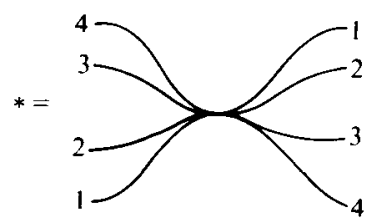

$$
(1,2,3,4) \stackrel{\leftrightarrow}{\longrightarrow}(4,3,2,1)
$$

\section{Figure 6}

The operations are illustrated in figure 7, where $\sim$ denotes two train tracks carrying the same foliations.

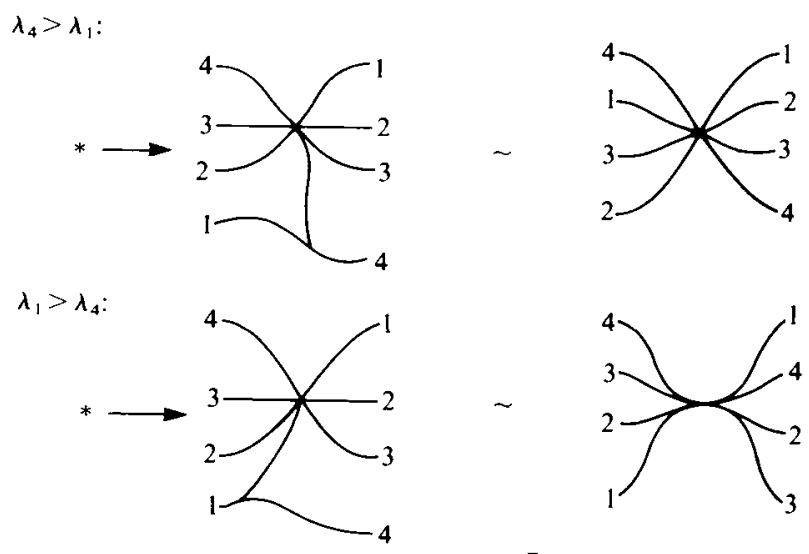

FIGURE 7

We leave the details to the interested reader. 


\section{REFERENCES}

[1] A. Hajian \& S. Kakutani. Weakly wandering sets and invariant measures. Trans. Amer. Math. Soc. 110 (1964), 136-151.

[2] M. Keane. Non-ergodic interval exchange transformations. Israel J. Math. 26 (1977), 188-196.

[3] S. P. Kerckhoff. The asymptotic geometry of Teichmüller space. Topology 19 (1980), 23-41.

[4] H. B. Keynes \& D. Newton. A minimal, non-uniquely ergodic interval exchange transformation. Math. Z. 148 (1976), 101-105.

[5] H. Masur. Interval exchange transformations and measured foliations. Ann. Math. 115 (1982), 169-200.

[6] G. Rauzy. Echanges d'intervalles et transformations induites. Acta Arith. 34 (1979), 315-328.

[7] M. Rees. An alternative approach to the ergodic theory of measured foliations on surfaces. Ergod. Th. \& Dynam. Sys. 1 (1981), 461-488.

[8] W. P. Thurston. The Geometry and Topology of Three-Manifolds, Chapter 9. Princeton Univ. notes.

[9] W. P. Thurston. On the geometry and dynamics of diffeomorphisms of surfaces. Preprint.

[10] W. Veech. Interval exchange transformations. J. Analyse Math. 33 (1978), 222-272.

[11] W. Veech. Projective Swiss cheeses and uniquely ergodic interval exchange transformations. In Progress in Mathematics, Vol. I. Birkhauser: Boston (1981), (pp 113-193).

[12] W. Veech. Gauss measures for transformations on the space of interval exchange maps. Ann. Math. 115 (1982), 201-242. 